\title{
Fibroblast Growth Factor 23 (FGF23) and Disorders of Phosphate Metabolism
}

\author{
Tasuku Saito $^{1}$ and Seiji Fukumoto ${ }^{2}$ \\ ${ }^{1}$ Department of Pediatrics, University of Tokyo Hospital, 7-3-1 Hongo, Bunkyo-ku, Tokyo 113-8655, Japan \\ ${ }^{2}$ Division of Nephrology \& Endocrinology, Department of Medicine, University of Tokyo Hospital, 7-3-1 Hongo, Bunkyo-ku, \\ Tokyo 113-8655, Japan
}

Correspondence should be addressed to Seiji Fukumoto, fukumoto-tky@umin.ac.jp

Received 28 May 2009; Accepted 27 July 2009

Recommended by Ali S Calikoglu

Derangements in serum phosphate level result in rickets/osteomalacia or ectopic calcification indicating that healthy people without these abnormalities maintain serum phosphate within certain ranges. These results indicate that there must be a regulatory mechanism of serum phosphate level. Fibroblast growth factor 23 (FGF23) was identified as the last member of FGF family. FGF23 is produced by bone and reduces serum phosphate level by suppressing phosphate reabsorption in proximal tubules and intestinal phosphate absorption through lowering 1,25-dihydroxyvitamin D level. It has been shown that excess and deficient actions of FGF23 result in hypophosphatemic rickets/osteomalacia and hyperphosphatemic tumoral calcinosis, respectively. These results indicate that FGF23 works as a hormone, and several disorders of phosphate metabolism can be viewed as endocrine diseases. It may become possible to treat patients with abnormal phosphate metabolism by pharmacologically modifying the activity of FGF23.

Copyright ( 2009 T. Saito and S. Fukumoto. This is an open access article distributed under the Creative Commons Attribution License, which permits unrestricted use, distribution, and reproduction in any medium, provided the original work is properly cited.

\section{Introduction}

It is well known that serum calcium $(\mathrm{Ca})$ level is regulated within a narrow range by actions of two calciumregulating hormones, parathyroid hormone (PTH) and 1,25-dihydroxyvitamin $\mathrm{D}\left[1,25(\mathrm{OH})_{2} \mathrm{D}\right]$. In contrast, while derangements in serum phosphate level result in rickets/osteomalacia or ectopic calcification, the regulatory mechanisms of serum phosphate have been largely unknown. Because PTH and $1,25(\mathrm{OH})_{2} \mathrm{D}$ can affect serum phosphate level, it has been unclear whether there is a tight mechanism of serum phosphate level regulated by a specific phosphateregulating hormone. However, the identification of fibroblast growth factor 23 (FGF23) and subsequent studies certainly changed this view. FGF23 works as a phosphate-regulating hormone and aberrant functions of FGF23 result in several diseases. Here, we briefly review the physiological and pathophysiological roles of FGF23.

\section{Structure and Function of FGF23}

FGF family members are now defined as humoral factorswhich have FGF homology region characterized by $\beta$-trefoil structure. FGF23 was identified as the last member of FGF family [1] and belongs to the FGF19 subfamily as well as FGF19 and FGF21 [2]. FGF23 is produced as a peptide with 251 amino acids by bone $[3,4]$. There is a signal peptide with 24 amino acids, and the secreted FGF23 protein consists of 227 amino acids which is approximately $32-\mathrm{kD}$ [5]. A part of FGF23 protein is proteolytically cleaved between ${ }^{179} \mathrm{Arg}$ and ${ }^{180}$ Ser into inactive fragments by enzymes that recognize ${ }^{176} \mathrm{R}-{ }^{177} \mathrm{X}-{ }^{178} \mathrm{X}-{ }^{179} \mathrm{R}$ motif just before the processing site (Figure 1). FGF homology region is present in the $\mathrm{N}$ terminal portion of this processing site of FGF23.

It has been shown that FGF23 suppresses the expression of type $2 \mathrm{a}$ and $2 \mathrm{c}$ sodium-phosphate cotransporters in the brush border membrane of proximal tubules which 
mediate physiological phosphate reabsorption. In addition, FGF23 reduces serum $1,25(\mathrm{OH})_{2} \mathrm{D}$ level by suppressing the expression of 25 -hydroxyvitamin $\mathrm{D}[25(\mathrm{OH}) \mathrm{D}]-1 \alpha-$ hydroxylase and also enhancing the expression of $25(\mathrm{OH}) \mathrm{D}-$ 24 -hydroxylase [6]. This $1 \alpha$-hydroxylase mediates the production of $1,25(\mathrm{OH})_{2} \mathrm{D}$ from $25(\mathrm{OH}) \mathrm{D}$, and 24-hydroxylase converts $1,25(\mathrm{OH})_{2} \mathrm{D}$ into more hydrophilic metabolites with less activity. Because $1,25(\mathrm{OH})_{2} \mathrm{D}$ enhances intestinal phosphate absorption, FGF23 reduces serum phosphate level by its suppressive effects on phosphate reabsorption in proximal tubules and phosphate absorption in intestine (Figure 2). Conversely, $1,25(\mathrm{OH})_{2} \mathrm{D}$ was shown to increase FGF23 level and enhance promoter activity of FGF23 gene [7]. It has been also shown that high phosphate diet increases circulatory FGF23 [8]. However, acute changes of serum phosphate do not alter FGF23 levels in human [9]. It remains to be clarified how changes in dietary phosphate modulate FGF23 levels.

While it is well known that PTH also decreases serum phosphate level by reducing the expression of type $2 a$ and $2 c$ sodium-phosphate cotransporters, effects of FGF23 on serum phosphate can be observed in thyroparathyroidectomized rats [6]. Therefore, while both PTH and FGF23 reduce the expression of sodium-phosphate cotransporters, FGF23 does not require PTH for its activity to reduce the expression of sodium-phosphate cotransporters. Although full-length FGF23 can induce hypophosphatemia, cleaved Nterminal and C-terminal fragments do not reduce serum phosphate level when injected into mice [10]. Therefore, only the full-length FGF23 has the biological activity to reduce serum phosphate level.

\section{FGF23 and Klotho}

FGF23 is produced by bone and acts on kidney indicating that FGF23 is a systemic factor in contrast to many other members of FGF family. It is well known that FGF family members bind to FGF receptors (FGFRs). There are four FGFR genes, and several FGFR subtypes are created form these genes by alternative splicing. However, the affinity of FGF23 to these FGFRs is low [11] suggesting that some other molecule(s) may be involved in FGF23 signaling. Indeed, FGF23 was found to bind to Klotho, a cell surface protein with homologies to $\beta$-glucosidases [11]. Klotho was originally identified as a gene product whose expression is severely reduced in transgenic mice named Klotho [12]. The evidence for Klotho's role in FGF23 action is as follows: (1) Klotho is localized in kidney, parathyroid gland, pituitary, and choroid plexus in the brain [13], the tissues where the inductions of early growth response (Egr)-1 expression and phosphorylation of extracellular signal-regulated kinase (ERK) were observed following FGF23 injections in mice [11]; (2) FGF23-null and Klotho mice show similar phenotypes such as hyperphosphatemia, inappropriately high levels of $1,25(\mathrm{OH})_{2} \mathrm{D}$ in the presence of hyperphosphatemia, calcifications of soft tissues, and short life span; (3) anti-Klotho antibody increases serum phosphate and $1,25(\mathrm{OH})_{2} \mathrm{D}$ levels in wild-type mice [11]; (4) circulatory

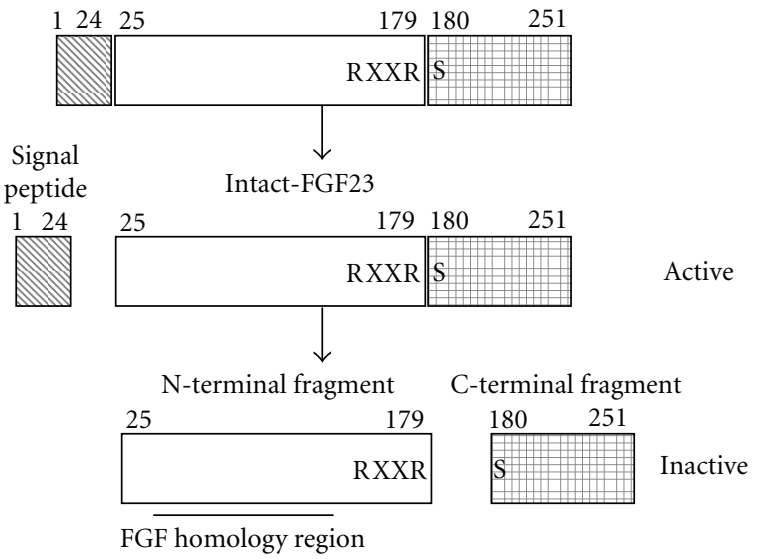

FIGURE 1: Structure FGF23 protein. FGF23 is produced as a peptide with 251 amino acids. There is a signal peptide with 24 amino acids, and the secreted FGF23 protein consists of 227 amino acids. A part of FGF23 protein is proteolytically cleaved between ${ }^{179} \mathrm{Arg}$ and ${ }^{180}$ Ser into inactive fragments by enzymes that recognize ${ }^{176} \mathrm{R}$ ${ }^{177} \mathrm{X}^{-178} \mathrm{X}-{ }^{179} \mathrm{R}$ motif just before the processing site. FGF homology region is present in the $\mathrm{N}$-terminal portion of this processing site of FGF23.

FGF23 level is extremely high in Klotho mice. Further studies have shown that Klotho, FGF23, and FGFR1c make a heterotrimer complex in vitro, and the binding of FGF23 to FGFR1c is enhanced by Klotho [11, 14]. Therefore, Klotho appears to be necessary for FGF23 to decrease serum phosphate and $1,25(\mathrm{OH})_{2} \mathrm{D}$ levels and is an indispensable molecule for FGF23 signaling (Figure 3).

\section{FGF23-Related Diseases}

4.1. Hypophosphatemic Diseases. Rickets and osteomalacia are characterized by impaired mineralization of bone matrix. Rickets develops in children before the closure of growth plate. Growth retardation and bone deformity are predominant features of rickets while severe muscle weakness and bone pain are usual symptoms of osteomalacia in adults. There are many causes of rickets and osteomalacia such as vitamin $\mathrm{D}$ deficiency, abnormal metabolism of vitamin $\mathrm{D}$, and renal tubular dysfunction (Table 1). Chronic hypophosphatemia is present in almost all cases of rickets and osteomalacia except for hypophosphatasia caused by mutations in TNALP gene encoding tissue nonspecific alkaline phosphatase [15]. It has been known that there are several kinds of hypophosphatemic rickets/osteomalacia with very similar clinical features [16]. These include autosomal dominant and recessive hypophosphatemic rickets/osteomalacia (ADHR, ARHR), X-linked hypophosphatemic rickets/osteomalacia (XLH), hypophosphatemic rickets/osteomalacia associated with McCune-Albright syndrome (MAS)/fibrous dysplasia (FD), and tumor-induced rickets/osteomalacia (TIO). These diseases are characterized by impaired phosphate reabsorption in proximal tubules. In addition, hypophosphatemia usually causes enhanced production of $1,25(\mathrm{OH})_{2} \mathrm{D}$ in 


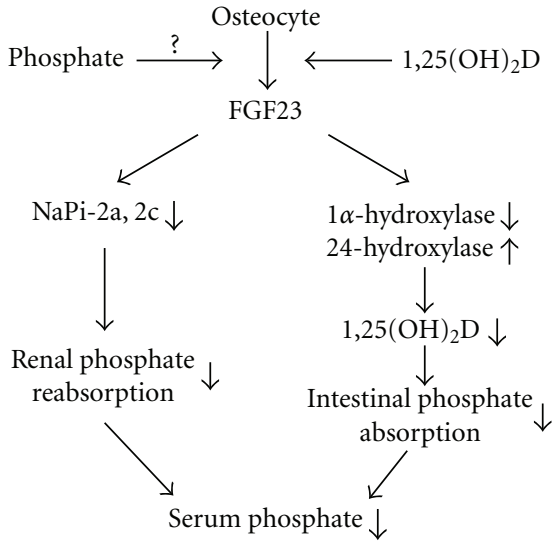

Figure 2: Actions of FGF23. FGF23 is produced by bone. FGF23 suppresses the expression of type $2 \mathrm{a}$ and $2 \mathrm{c}$ sodium-phosphate cotransporters $(\mathrm{NaPi})$ in the brush border membrane of proximal tubules which mediate physiological phosphate reabsorption. In addition, FGF23 reduces serum $1,25(\mathrm{OH})_{2} \mathrm{D}$ level by suppressing the expression of 25 -hydroxyvitamin $\mathrm{D}[25(\mathrm{OH}) \mathrm{D}]-1 \alpha$-hydroxylase and also enhancing the expression of $25(\mathrm{OH}) \mathrm{D}-24$-hydroxylase. This $1 \alpha$-hydroxylase mediates the production of $1,25(\mathrm{OH})_{2} \mathrm{D}$ from $25(\mathrm{OH}) \mathrm{D}$, and 24-hydroxylase converts $1,25(\mathrm{OH})_{2} \mathrm{D}$ into more hydrophilic metabolites with less activity. Because $1,25(\mathrm{OH})_{2} \mathrm{D}$ enhances intestinal phosphate absorption, FGF23 reduces serum phosphate level by its suppressive effects on renal phosphate reabsorption and intestinal phosphate absorption. Conversely, $1,25(\mathrm{OH})_{2} \mathrm{D}$ increases FGF23 level. High phosphate diet also increases circulatory FGF23. However, it remains to be clarified how changes in dietary phosphate modulate FGF23 level.

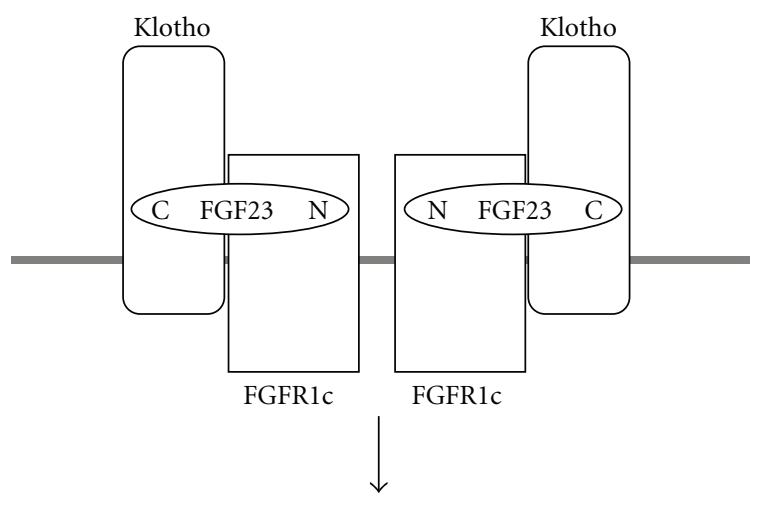

Signal transduction

Figure 3: Heterotrimer complex of Klotho, FGF23, and FGFR1c. Klotho, FGF23, and FGFR1c make a heterotrimer complex in order to transmit the signal of FGF23 to target organs. Therefore, Klotho seems to be necessary for FGF23 signaling. N: N-terminus of FGF23 protein; C: C-terminus of FGF23 protein.

proximal tubules and increases serum $1,25(\mathrm{OH})_{2} \mathrm{D}$ level. However, serum $1,25(\mathrm{OH})_{2} \mathrm{D}$ levels in these FGF23-related hypophosphatemic rickets/osteomalacia remain low to low normal. Therefore, it was presumed that not only impaired proximal tubular phosphate reabsorption but also abnormal metabolism of vitamin D underlie these hypophosphatemic diseases. As easily expected from actions of FGF23, it has been shown that FGF23 is closely related to these hypophosphatemic diseases. In addition to these diseases, it has been recently reported that infusion of saccharated ferric oxide (iron polymaltose) can also lead to renal phosphate wasting mediated by increased FGF23 [17-19].

4.2. ADHR. ADHR is a rare familial hypophosphatemic rickets/osteomalacia which does not respond to physiological dose of native vitamin D. FGF23 was identified as a responsible gene for ADHR by positional cloning in 2000 [20]. Three heterozygous missense mutations around the processing site of FGF23 protein have been identified in ADHR families. These mutations replace ${ }^{176} \mathrm{Arg}$ or ${ }^{179} \mathrm{Arg}$ in FGF23 protein with other amino acids destroying R-X-X-R motif. Therefore, it has been presumed that the cleavage of FGF23 protein between ${ }^{179} \mathrm{Arg}$ and ${ }^{180}$ Ser is prevented by these mutations causing increased full-length FGF23 level. However, the circulatory FGF23 levels in $42 \mathrm{ADHR}$ patients were not significantly different from those of controls [21]. On the other hand, FGF23 levels in ADHR patients fluctuate with time and are high when they show hypophosphatemia. These results indicate that FGF23 levels are not always high in patients with ADHR indicating that the resistance to the processing of FGF23 protein alone does not explain enhanced activity of FGF23 in these patients. We have previously shown that FGF23 levels are low in hypophosphatemic patients caused by other etiologies than FGF23 excess such as Fanconi syndrome and vitamin D deficiency [22]. Therefore, high FGF23 levels in the presence of hypophosphatemia in patients with ADHR rather suggest that the regulatory mechanisms of FGF23 production are somehow deranged in these patients. Further studies are necessary to clarify the pathogenesis of hypophosphatemia in ADHR patients.

4.3. ARHR. ARHR is also a rare familial hypophosphatemic rickets/osteomalacia which shows resistance to native vitamin D like ADHR. Almost all cases are observed in families with consanguineous marriage. Dentin matrix protein $(D M P) 1$ was identified as a responsible gene for ARHR by positional cloning in 2006, and several homozygous mutations in DMP1 gene were identified in patients with ARHR $[23,24]$. DMP1 is a matrix protein found in osteocytes and odontoblasts and belongs to a family of small integrinbinding ligand, N-linked glycoproteins (SIBLING) together with matrix proteins in calcified tissues such as dentin sialophosphoprotein (DSPP), integrin-binding sialoprotein (IBSP), matrix extracellular phosphoglycoprotein (MEPE), and osteopontin. It is reported that homozygous DMP1 knock-out mice show features of hypophosphatemic rickets, and serum FGF23 levels of DMP1 knock-out mice and ARHR patients are high [23, 24]. In addition, FGF23 was shown to be abundantly expressed in osteocytes of DMP1-null mice. Therefore, excess production of FGF23 in osteocytes seems to cause ARHR. However, it remains unclear how mutations in DMP1 gene cause enhanced production of FGF23. 
TABLE 1: Causes of rickets/osteomalacia.

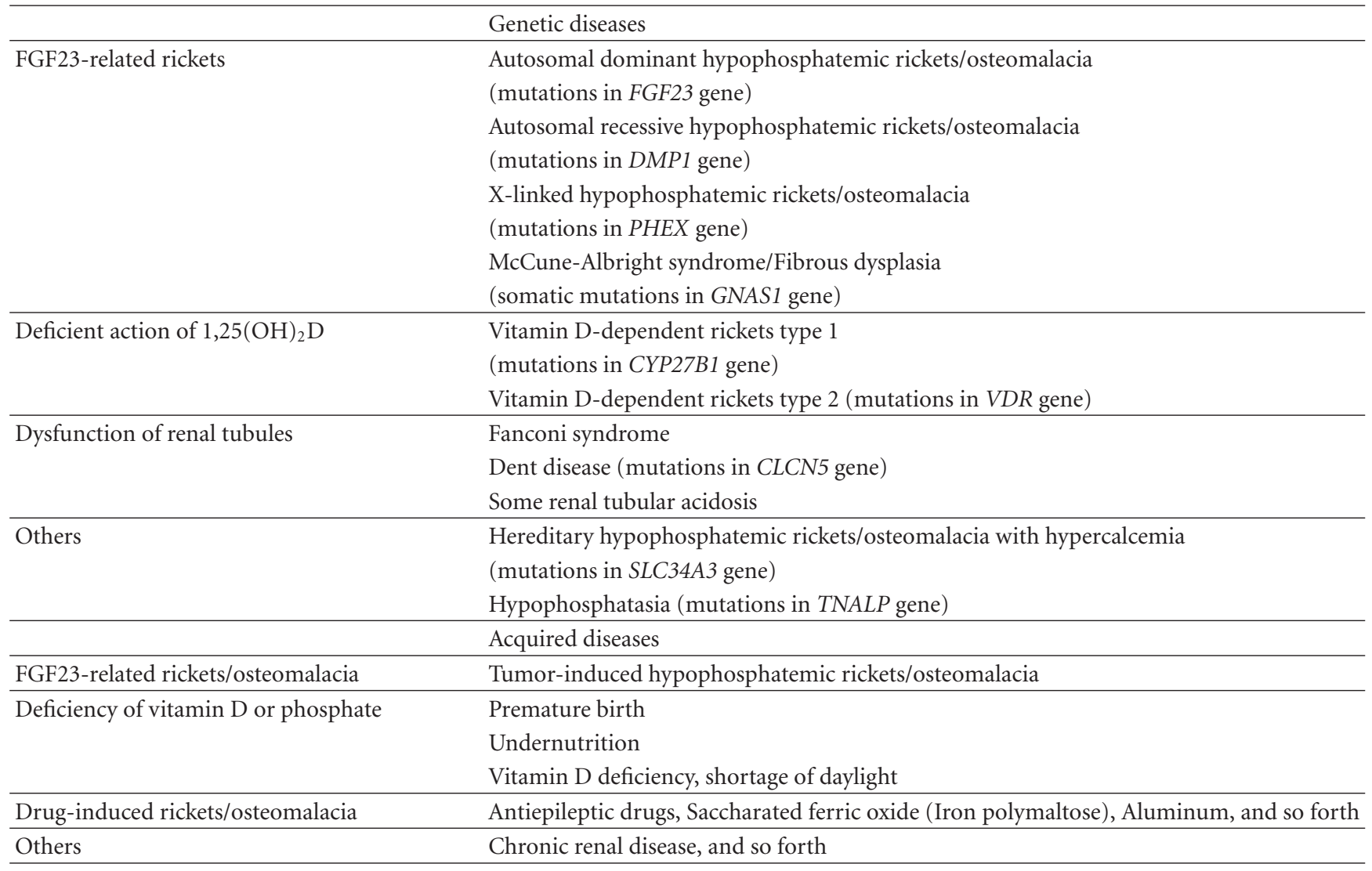

FGF23: fibroblast growth factor 23; DMP1: dentin matrix protein 1; PHEX: phosphate-regulating gene with homologies to endopeptidases on the X chromosome; VDR: vitamin D receptor; GNAS1: guanine nucleotide binding protein, alpha stimulating 1; TNALP: Tissue nonspecific alkaline phosphatase.

4.4. XLH. XLH is considered to be the most frequent cause of vitamin D-resistant hypophosphatemic rickets/osteomalacia. The frequency of XLH is reported to be about 1 in 20000 births [25]. The responsible gene for XLH was identified in 1995 and named phosphate-regulating gene with homologies to endopeptidases on the $X$ chromosome (PHEX) [26]. The expression of PHEX is found in osteocytes, osteoblasts, and odontoblasts [27]. Although PHEX protein shows homology to endopeptidases with single membrane-spanning region, it is not clear whether PHEX physiologically works as an endopeptidase. Hyp mouse, which has a deletion in $3^{\prime}$ portion of Phex gene, is known as a model of XLH. Several results suggest that hypophosphatemia in Hyp and XLH patients is caused by some humoral factor. For example, the crosstransplantaion of kidneys in wild-type and Hyp mice did not change their phenotypes [28]. In addition, renal transplantation from a healthy donor to a patient with XLH did not correct renal phosphate wasting [29]. It has been shown that serum FGF23 levels in most XLH patients are above the reference range $[30,31]$. Serum FGF23 levels in Hyp mice are also elevated, and excess production of FGF23 is found particularly in bone of Hyp mice. These results indicate that the overexpression of FGF23 in bone is responsible for hypophosphatemic rickets/osteomalacia in patients with XLH and Hyp mice. Again, it remains to be clarified how PHEX protein regulates the synthesis of FGF23 in bone.

4.5. MAS/FD. FD is a bone lesion in which medullary cavity is replaced by fibrous, osseous, and chondral tissues. FD occurs either as monostotic $(70 \%-80 \%)$ or as polyostotic $(20 \%-30 \%)$ form. MAS is a syndrome consisting of polyostotic fibrous dysplasia, skin hyperpigmentation (café-aulait spots), and endocrine dysfunction, frequently seen in females as precocious puberty. MAS is caused by somatic mosaicism of cells harboring activating mutations in guanine nucleotide binding protein, alpha stimulating 1 (GNAS1) gene. These mutations are also observed in FD tissues without MAS. Approximately $50 \%$ of MAS/FD patients show hypophosphatemic rickets/osteomalacia. It was reported that FGF23 production is found in bone including regions of FD, and circulatory FGF23 levels are increased in MAS/FD patients who show hypophosphatemic rickets/osteomalacia [3]. However, it is not demonstrated that enhanced cyclic AMP level actually increases FGF23 production, and the mechanism of FGF23 overproduction remains to be clarified.

4.6. TIO. TIO is a paraneoplastic syndrome usually associated with mesenchymal slow-growing tumors. Most tumors 
responsible for TIO are now pathologically classified as phosphaturic mesenchymal tumors, mixed connective tissue variant (PMTMCT). FGF23 was identified as a causative humoral factor for TIO, which is quite rare in childhood [5]. FGF23 was shown to be abundantly expressed in tumors causing TIO $[5,32]$. Circulatory FGF23 levels are elevated in virtually all patients with TIO [31]. The surgical removal of responsible tumors results in normalization of FGF23 levels and cures this disease.

\section{Hyperphosphatemic Diseases}

Tumoral calcinosis is characterized by ectopic calcification especially around large joints. While the commonest form of tumoral calcinosis is observed in patients with endstage renal disease undergoing dialysis, there are hereditary forms of tumoral calcinosis with normal renal function $[33,34]$. Familial hyperphosphatemic tumoral calcinosis is a rare autosomal recessive disorder that is characterized by enhanced renal tubular phosphate reabsorption and rather high serum $1,25(\mathrm{OH})_{2} \mathrm{D}$ levels for hyperphosphatemia [34].

Biallelic mutations in GALNT3, FGF23, and Klotho have been shown to cause this hyperphosphatemic disease [35-38]. Mutations in GALNT3 and FGF23 have been shown to cause susceptibility of FGF23 protein to the processing between ${ }^{179} \mathrm{Arg}$ and ${ }^{180} \mathrm{Ser}$ resulting in low levels of full-length FGF23. GALNT3 encodes a protein called UDP-N-acetyl-alpha-D-galactosamine: polypeptide $\mathrm{N}$-acetylgalactosaminyltransferase 3 . This is an enzyme involved in the synthesis of mutin-type O-linked glycans. Homozygous missense mutations in GALNT3 gene seem to cause impaired glycosylation of FGF23 protein making it susceptible for the processing. FGF23 mutations are postulated to cause the susceptibility by changing protein structure of FGF23. A homozygous mutation of Klotho has been shown to make the expression of Klotho protein to be markedly reduced, resulting in diminished ability of FGF23 protein to act on target organs [38].

FGF23 is marginally elevated in patients with hypoparathyroidism [39]. High level of FGF23 is also found in patients with chronic kidney disease, especially in patients with end-stage renal disease [40]. The high level of FGF23 in these diseases is regarded as a compensatory response to hyperphosphatemia or phosphate overload. However, it is still possible that some other factors associated with impaired renal function are contributing to the increase of FGF23 because FGF23 level is extremely high in some patients with end-stage renal disease.

\section{Conclusion}

FGF23 was shown to be produced by bone and act on kidney through a specific receptor system. In addition, excess and deficient actions of FGF23 result in hypophosphatemic and hyperphosphatemic diseases, respectively. These results indicate that FGF23 works as a hormone, and several disorders of phosphate metabolism can be viewed as endocrine diseases. However, there still remain several important questions unanswered such as regulatory mechanisms of FGF23 production and signals beyond Klotho-FGFR1c that mediate FGF23 action. On the other hand, anti-FGF23 antibodies were already shown to increase serum phosphate and $1,25(\mathrm{OH})_{2} \mathrm{D}$ levels both in wild-type and Hyp mice [41, 42]. Like many other endocrine diseases, it may become possible to treat patients with abnormal phosphate metabolism by regulating the activity of this phosphotropic hormone.

\section{Acknowledgments}

This work was partly supported by Grants from Ministry of Education, Culture, Sports, Science and Technology of Japan and from Ministry of Health, Labor and Welfare of Japan.

\section{References}

[1] T. Yamashita, M. Yoshioka, and N. Itoh, "Identification of a novel fibroblast growth factor, FGF-23, preferentially expressed in the ventrolateral thalamic nucleus of the brain," Biochemical and Biophysical Research Communications, vol. 277, no. 2, pp. 494-498, 2000.

[2] N. Itoh and D. M. Ornitz, "Evolution of the FGF and FGFr gene families," Trends in Genetics, vol. 20, no. 11, pp. 563-569, 2004.

[3] M. Riminucci, M. T. Collins, N. S. Fedarko, et al., "FGF23 in fibrous dysplasia of bone and its relationship to renal phosphate wasting," Journal of Clinical Investigation, vol. 112, no. 5, pp. 683-692, 2003.

[4] D. Sitara, M. S. Razzaque, M. Hesse, et al., "Homozygous ablation of fibroblast growth factor-23 results in hyperphosphatemia and impaired skeletogenesis, and reverses hypophosphatemia in Phex-deficient mice," Matrix Biology, vol. 23, no. 7, pp. 421-432, 2004.

[5] T. Shimada, S. Mizutani, T. Muto, et al., "Cloning and characterization of FGF23 as a causative factor of tumorinduced osteomalacia," Proceedings of the National Academy of Sciences of the United States of America, vol. 98, no. 11, pp. 6500-6505, 2001.

[6] T. Shimada, H. Hasegawa, Y. Yamazaki, et al., "FGF-23 is a potent regulator of vitamin D metabolism and phosphate homeostasis," Journal of Bone and Mineral Research, vol. 19, no. 3, pp. 429-435, 2004.

[7] S. Liu, W. Tang, J. Zhou, et al., "Fibroblast growth factor 23 is a counter-regulatory phosphaturic hormone for vitamin D," Journal of the American Society of Nephrology, vol. 17, no. 5, pp. 1305-1315, 2006.

[8] T. Shimada, Y. Yamazaki, M. Takahashi, et al., "Vitamin D receptor-independent FGF23 actions in regulating phosphate and vitamin D metabolism," American Journal of Physiology, vol. 289, no. 5, pp. 1088-1095, 2005.

[9] N. Ito, S. Fukumoto, Y. Takeuchi, et al., "Effect of acute changes of serum phosphate on fibroblast growth factor (FGF)23 levels in humans," Journal of Bone and Mineral Metabolism, vol. 25, no. 6, pp. 419-422, 2007.

[10] T. Shimada, T. Muto, I. Urakawa, et al., "Mutant FGF-23 responsible for autosomal dominant hypophosphatemic rickets is resistant to proteolytic cleavage and causes hypophosphatemia in vivo," Endocrinology, vol. 143, no. 8, pp. 31793182, 2002. 
[11] I. Urakawa, Y. Yamazaki, T. Shimada, et al., "Klotho converts canonical FGF receptor into a specific receptor for FGF23," Nature, vol. 444, no. 7120, pp. 770-774, 2006.

[12] M. Kuro-o, Y. Matsumura, H. Aizawa, et al., "Mutation of the mouse klotho gene leads to a syndrome resembling ageing," Nature, vol. 390, no. 6655, pp. 45-51, 1997.

[13] S. A. Li, M. Watanabe, H. Yamada, et al., "Immunohistochemical localization of Klotho protein in brain, kidney, and reproductive organs of mice," Cell Structure and Function, vol. 29, no. 4, pp. 91-99, 2004.

[14] H. Kurosu, Y. Ogawa, M. Miyoshi, et al., "Regulation of fibroblast growth factor-23 signaling by Klotho," Journal of Biological Chemistry, vol. 281, no. 10, pp. 6120-6123, 2006.

[15] M. D. Ruppe and S. M. Jan de Beur, "Disorders of phosphate homeostasis," in Primer on the Metabolic Bone Diseases and Disorders of Mineral Metabolism, C. J. Rosen, Ed., pp. 317-325, America Society for Bone and Mineral Research, Washington, DC,USA, 7th edition.

[16] S. Liu, J. Zhou, W. Tang, R. Menard, J. Q. Feng, and L. D. Quarles, "Pathogenic role of FGF23 in Dmp1-null mice," American Journal of Physiology, vol. 295, no. 2, pp. 254-261, 2008.

[17] B. J. Schouten, M. P. Doogue, S. G. Soule, et al., "Iron polymaltose-induced FGF23 elevation complicated by hypophosphataemic osteomalacia," Annals of Clinical Biochemistry, vol. 46, no. 2, pp. 167-169, 2009.

[18] B. J. Schouten, P. J. Hunt, J. H. Livesey, et al., "FGF23 elevation and hypophosphatemia after intravenous iron polymaltosea prospective study," Journal of Clinical Endocrinology and Metabolism, vol. 94, no. 7, pp. 2332-2337, 2009.

[19] Y. Shimizu, Y. Tada, M. Yamauchi, et al., "Hypophosphatemia induced by intravenous administration of saccharated ferric oxide: another form of FGF23-releted hypophosphatemia," Bone, vol. 45, no. 4, pp. 814-816, 2009.

[20] ADHR Consortium, "Autosomal dominant hypophosphatemic rickets is associated with mutations in FGF23," Nature Genetics, vol. 26, no. 3, pp. 345-348, 2000.

[21] E. A. Imel, S. L. Hui, M. J. Econs, et al., "FGF23 concentrations vary with disease status in autosomal dominant hypophosphatemic rickets," Journal of Bone and Mineral Research, vol. 22, no. 4, pp. 520-526, 2007.

[22] I. Endo, S. Fukumoto, K. Ozono, et al., "Clinical usefulness of measurement of fibroblast growth factor 23 (FGF23) in hypophosphatemic patients: proposal of diagnostic criteria using FGF23 measurement," Bone, vol. 42, no. 6, pp. 12351239, 2008.

[23] B. Lorenz-Depiereux, M. Bastepe, A. Benet-Pages, et al., "DMP1 mutations in autosomal recessive hypophosphatemia implicate a bone matrix protein in the regulation of phosphate homeostasis," Nature Genetics, vol. 38, no. 11, pp. 1248-1250, 2006.

[24] J. Q. Feng, L. M. Ward, S. Liu, et al., "Loss of DMP1 causes rickets and osteomalacia and identifies a role for osteocytes in mineral metabolism," Nature Genetics, vol. 38, no. 11, pp. 1310-1315, 2006.

[25] H. S. Tenenhouse, "X-linked hypophosphataemia: a homologous disorder in humans and mice," Nephrology Dialysis Transplantation, vol. 14, no. 2, pp. 333-341, 1999.

[26] The HYP Consortium, "A gene (PEX) with homologies to endopeptidases is mutated in patients with X-linked hypophosphatemic rickets," Nature Genetics, vol. 11, no. 2, pp. 130-136, 1995.

[27] L. Beck, Y. Soumounou, J. Martel, et al., "Pex/PEX tissue distribution and evidence for a deletion in the $3^{\prime}$ region of the Pex gene in X-linked hypophosphatemic mice," Journal of Clinical Investigation, vol. 99, no. 6, pp. 1200-1209, 1997.

[28] T. Nesbitt, T. M. Coffman, R. Griffiths, et al., "Crosstransplantation of kidneys in normal and Hyp mice. Evidence that the Hyp mouse phenotype is unrelated to an intrinsic renal defect," Journal of Clinical Investigation, vol. 89, no. 5, pp. 1453-1459, 1992.

[29] J. M. Morgan, W. L. Hawley, A. I. Chenoweth, et al., "Renal transplantation in hypophosphatemia with vitamin D resistant rickets," Archives of Internal Medicine, vol. 134, no. 3, pp. 549-552, 1974.

[30] Y. Yamazaki, R. Okazaki, M. Shibata, et al., "Increased circulatory level of biologically active full-length FGF-23 in patients with hypophosphatemic rickets/osteomalacia," Journal of Clinical Endocrinology and Metabolism, vol. 87, no. 11, pp. 4957-4960, 2002.

[31] N. Ito, S. Fukumoto, Y. Takeuchi, et al., "Comparison of two assays for fibroblast growth factor (FGF)-23," Journal of Bone and Mineral Metabolism, vol. 23, no. 6, pp. 435-440, 2005.

[32] K. E. White, K. B. Jonsson, G. Carn, et al., "The autosomal dominant hypophosphatemic rickets (ADHR) gene is a secreted polypeptide overexpressed by tumors that cause phosphate wasting," Journal of Clinical Endocrinology and Metabolism, vol. 86, no. 2, pp. 497-500, 2001.

[33] O. Topaz, M. Indelman, I. Chefetz, et al., "A deleterious mutation in SAMD9 causes normophosphatemic familial tumoral calcinosis," American Journal of Human Genetics, vol. 79, no. 4, pp. 759-764, 2006.

[34] K. W. Lyles, D. L. Halsey, N. E. Friedman, et al., "Correlations of serum concentrations of 1,25-dihydroxyvitamin D, phosphorus, and parathyroid hormone in tumoral calcinosis," Journal of Clinical Endocrinology and Metabolism, vol. 67, no. 1, pp. 88-92, 1988.

[35] O. Topaz, D. L. Shurman, R. Bergman, et al., "Mutations in GALNT3, encoding a protein involved in O-linked glycosylation, cause familial tumoral calcinosis," Nature Genetics, vol. 36, no. 6, pp. 579-581, 2004.

[36] T. Larsson, S. I. Davis, H. J. Garringer, et al., "FGF23 mutants causing familial tumoral calcinosis are differentially processed," Endocrinology, vol. 146, no. 9, pp. 3883-3891, 2005.

[37] K. Araya, S. Fukumoto, R. Backenroth, et al., "A novel mutation in fibroblast growth factor 23 gene as a cause of tumoral calcinosis," Journal of Clinical Endocrinology and Metabolism, vol. 90, no. 10, pp. 5523-5527, 2005.

[38] S. Ichikawa, E. A. Imel, M. L. Kreiter, et al., "A homozygous missense mutation in human KLOTHO causes severe tumoral calcinosis," Journal of Clinical Investigation, vol. 117, no. 9, pp. 2684-2691, 2007.

[39] A. Gupta, K. Winer, M. J. Econs, et al., "FGF-23 is elevated by chronic hyperphosphatemia," Journal of Clinical Endocrinology and Metabolism, vol. 89, no. 9, pp. 4489-4492, 2004.

[40] T. J. Weber, S. Liu, O. S. Indridason, et al., "Serum FGF23 levels in normal and disordered phosphorus homeostasis," Journal of Bone and Mineral Research, vol. 18, no. 7, pp. 1227-1234, 2003.

[41] Y. Yamazaki, T. Tamada, N. Kasai, et al., "Anti-FGF23 neutralizing antibodies show the physiological role and structural features of FGF23," Journal of Bone and Mineral Research, vol. 23, no. 9, pp. 1509-1518, 2008.

[42] Y. Aono, Y. Yamazaki, J. Yasutake, et al., "Therapeutic effects of anti-FGF23 antibodies in hypophosphatemic rickets/osteomalacia," Journal of Bone and Mineral Research. In press. 